\title{
DIFFERENTIABILITY OF THE ARITHMETIC VOLUME FUNCTION
}

\author{
Huayi Chen
}

\begin{abstract}
We introduce the positive intersection product in Arakelov geometry and prove that the arithmetic volume function is continuously differentiable. As applications, we compute the distribution function of the asymptotic measure of a Hermitian line bundle and several other arithmetic invariants.

Résumé. - On introduit le produit d'intersection positive en géométrie d'Arakelov et on démontre que la fonction volume arithmétique est continuement dérivable. Comme applications, on calcule la fonction de répartition de la mesure de probabilité asymptotique d'un fibré inversible hermitien ainsi que quelques d'autres invariants arithmétiques.
\end{abstract}

\section{Contents}

1. Introduction ........................................

2. Notation and preliminaries...............................

3. Positive intersection product........................... 6

4. Differentiability of the arithmetic volume function........... 10

5. Applications and comparisons......................... 13

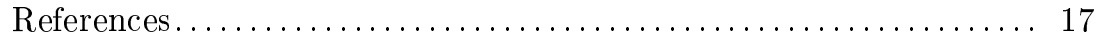

\section{Introduction}

Let $K$ be a number field, $\mathcal{O}_{K}$ be its integer ring and $\pi: X \rightarrow \operatorname{Spec} \mathcal{O}_{K}$ be an arithmetic variety of relative dimension $d$. Recall that the arithmetic volume of a continuous Hermitian line bundle $\bar{L}$ on $X$ is by definition

$$
\widehat{\operatorname{vol}}(\bar{L}):=\limsup _{n \rightarrow \infty} \frac{\widehat{h}^{0}\left(X, \bar{L}^{\otimes n}\right)}{n^{d+1} /(d+1) !}
$$

where

$$
\widehat{h}^{0}\left(X, \bar{L}^{\otimes n}\right)=\log \#\left\{s \in \pi_{*}\left(L^{\otimes n}\right) \mid \forall \sigma: K \rightarrow \mathbb{C},\|s\|_{\sigma, \text { sup }} \leqslant 1\right\} .
$$


The properties of the arithmetic volume $\widehat{\text { vol }}$ (see $[\mathbf{2 1}, \mathbf{2 2}, \mathbf{2 6},[\mathbf{2 7}, \mathbf{1 1}, \widehat{\mathbf{1 2}}]$ ) are quite similar to the corresponding properties of the classical volume function in algebraic geometry. Recall that if $Y$ is a projective variety defined over a field $k$ and if $L$ is a line bundle on $Y$, then the volume of $L$ is defined as

$$
\operatorname{vol}(L):=\limsup _{n \rightarrow \infty} \frac{\operatorname{rk}_{k} H^{0}\left(Y, L^{\otimes n}\right)}{n^{\operatorname{dim} Y} /(\operatorname{dim} Y) !} .
$$

In [8], Boucksom, Favre and Jonsson have been interested in the regularity of the geometric volume function. They have actually proved that the function $\operatorname{vol}(L)$ is continuously differentiable on the big cone. The same result has also been independently obtained by Lazarsfeld and Musţată [18, by using Okounkov bodies. Note that the geometric volume function is not second order derivable in general, as shown by the blow up of $\mathbb{P}^{2}$ at a closed point, see [17, 2.2.46] for details. In the differential of vol appears the positive intersection product, initially defined in $[\mathbf{7}]$ in the analytic-geometrical framework, and redefined algebraically in [8].

Inspired by [8], we introduce an analogue of the positive intersection product in Arakelov geometry and prove that the arithmetic volume function $\widehat{\mathrm{vol}}$ is continuously differentiable on $\widehat{\operatorname{Pic}}(X)$. We shall establish the following theorem:

Theorem 1.1. - Let $\bar{L}$ and $\bar{M}$ be two continuous Hermitian line bundles on $X$. Assume that $\bar{L}$ is big. Then

$$
D_{\bar{L}} \widehat{\operatorname{vol}}(\bar{M}):=\lim _{n \rightarrow+\infty} \frac{\widehat{\operatorname{vol}}\left(\bar{L}^{\otimes n} \otimes \bar{M}\right)-\widehat{\operatorname{vol}}\left(\bar{L}^{\otimes n}\right)}{n^{d}}
$$

exists in $\mathbb{R}$, and the function $\widehat{D_{\bar{L}} \widehat{\mathrm{vol}}}$ is additive on $\widehat{\mathrm{Pic}}(X)$. Furthermore, one has

$$
D_{\bar{L}} \widehat{\operatorname{vol}}(\bar{M})=(d+1)\left\langle\widehat{c}_{1}(\bar{L})^{d}\right\rangle \cdot \widehat{c}_{1}(\bar{M}) .
$$

Here the positive intersection product $\left\langle\widehat{c}_{1}(\bar{L})^{d}\right\rangle$ is defined as the least upper bound of self intersections of ample Hermitian line bundles dominated by $\bar{L}$ (see $\$ 3.3$ infra). In particular, one has $\left\langle\widehat{c}_{1}(\bar{L})^{d}\right\rangle \cdot \widehat{c}_{1}(\bar{L})=\left\langle\widehat{c}_{1}(\bar{L})^{d+1}\right\rangle=\widehat{\operatorname{vol}}(\bar{L})$, which shows that the arithmetic Fujita approximation is asymptotically orthogonal.

As an application, we calculate explicitly the distribution function of the asymptotic measure (see [10, 11]) of a generically big Hermitian line bundle in terms of positive intersection numbers. Let $\bar{L}$ be a Hermitian line bundle on $X$ such that $L_{K}$ is big. The asymptotic measure $\nu_{\bar{L}}$ is the vague limit (when $n$ goes to infinity) of Borel probability measures whose distribution functions are determined by the filtration of $H^{0}\left(X_{K}, L_{K}^{\otimes n}\right)$ by successive minima (see (13) infra). Several asymptotic invariants can be obtained by integration with respect to $\nu_{\bar{L}}$. Therefore, it is interesting to determine completely the distribution of $\nu_{\bar{L}}$, which will be given in Proposition 5.2 by using the positive intersection product.

The article is organized as follows. In the second section, we recall some positivity conditions for Hermitian line bundles and discuss their properties. In the third section, we define the positive intersection product in Arakelov geometry. It is in the fourth section that we establish the differentiability of the arithmetic volume function. Finally in the fifth section, we present applications on the asymptotic measure and 
we compare our result to some known results on the differentiability of arithmetic invariants.

Acknowledgement: I would like to thank R. Berman, D. Bertrand, J.-B. Bost, S. Boucksom, C. Favre and V. Maillot for interesting and helpful discussions. I am also grateful to M. Jonsson for remarks.

\section{Notation and preliminaries}

In this article, we fix a number field $K$ and denote by $\mathcal{O}_{K}$ its integer ring. Let $\bar{K}$ be an algebraic closure of $K$. Let $\pi: X \rightarrow$ Spec $\mathcal{O}_{K}$ be a projective and flat morphism and $d$ be the relative dimension of $\pi$. Denote by $\widehat{\operatorname{Pic}}(X)$ the group of isomorphism classes of (continuous) Hermitian line bundles on $X$. If $\bar{L}$ is a Hermitian line bundle on $X$, we denote by $\pi_{*}(\bar{L})$ the $\mathcal{O}_{K}$-module $\pi_{*}(L)$ equipped with sup norms.

In the following, we recall several notions about Hermitian line bundles. The references are [16, 28, 6, 20].

Assume that $x \in X(\bar{K})$ is an algebraic point of $X$. Denote by $K_{x}$ the field of definition of $x$ and by $\mathcal{O}_{x}$ its integer ring. The morphism $x: \operatorname{Spec} \bar{K} \rightarrow X$ gives rise to a point $P_{x}$ of $X$ valued in $\mathcal{O}_{x}$. The pull-back of $\bar{L}$ by $P_{x}$ is a Hermitian line bundle on Spec $\mathcal{O}_{x}$. We denote by $h_{\bar{L}}(x)$ its normalized Arakelov degree, called the height of $x$. Note that the height function is additive with respect to $\bar{L}$.

Let $\bar{L}$ be a Hermitian line bundle on $X$. We say that a section $s \in \pi_{*}(L)$ is effective (resp. strictly effective) if for any $\sigma: K \rightarrow \mathbb{C}$, one has $\|s\|_{\sigma \text {,sup }} \leqslant 1$ (resp. $\left.\|s\|_{\sigma \text {,sup }}<1\right)$. We say that the Hermitian line bundle $\bar{L}$ is effective if it admits a non-zero effective section.

Let $\bar{L}_{1}$ and $\bar{L}_{2}$ be two Hermitian line bundles on $X$. We say that $\bar{L}_{1}$ is smaller than $\bar{L}_{2}$ and we denote by $\bar{L}_{1} \leqslant \bar{L}_{2}$ if the Hermitian line bundle $\bar{L}_{1}^{\vee} \otimes \bar{L}_{2}$ is effective.

We say that a Hermitian line bundle $\bar{A}$ is ample if $A$ is ample, $c_{1}(\bar{A})$ is semi-positive in the sense of current on $X(\mathbb{C})$ and $\widehat{c}_{1}\left(\left.\bar{L}\right|_{Y}\right)^{\operatorname{dim} Y}>0$ for any integral sub-scheme $Y$ of $X$ which is flat over $\operatorname{Spec} \mathcal{O}_{K}$. Here the intersection number $\widehat{c}_{1}\left(\left.\bar{L}\right|_{Y}\right)^{\operatorname{dim} Y}$ is defined in the sense of [28] (see Lemma 6.5 loc. cit., see also [29]). Note that there always exists an ample Hermitian line bundle on $X$. In fact, since $X$ is projective, it can be embedded in a projective space $\mathbb{P}^{N}$. Then the restriction of $\mathcal{O}_{\mathbb{P}^{N}}(1)$ with Fubini-Study metrics on $X$ is ample. Note that the Hermitian line bundle $\bar{L}$ thus constructed has strictly positive smooth metrics. Thus, if $\bar{M}$ is an arbitrary Hermitian line bundle with smooth metrics on $X$, then for sufficiently large $n, \bar{M} \otimes \bar{L}^{\otimes n}$ is still ample.

We say that a Hermitian line bundle $\bar{N}$ is vertically nef if the restriction of $N$ on each fiber of $\pi$ is nef and $c_{1}(\bar{N})$ is semi-positive in the sense of current on $X(\mathbb{C})$. We say that $\bar{N}$ is nef if it is vertically nef and $\widehat{c}_{1}\left(\left.\bar{N}\right|_{Y}\right) \operatorname{dim} Y \geqslant 0$ for any integral sub-scheme $Y$ of $X$ which is flat over $\operatorname{Spec} \mathcal{O}_{K}$. By definition, an ample Hermitian line bundle is always nef. Furthermore, if $\bar{A}$ is an ample Hermitian line bundle and if $\bar{N}$ is a Hermitian line bundle such that $\bar{N}^{\otimes n} \otimes \bar{A}$ is ample for any integer $n \geqslant 1$, then $\bar{N}$ is nef. We denote by $\widehat{\operatorname{Nef}}(X)$ the subgroup of $\widehat{\operatorname{Pic}}(X)$ consisting of nef Hermitian line bundles. 
If $f: X(\mathbb{C}) \rightarrow \mathbb{R}$ is a continuous function, we denote by $\overline{\mathcal{O}}(f)$ the Hermitian line bundle on $X$ whose underlying line bundle is trivial, and such that the norm of the unit section 1 at $x \in X(\mathbb{C})$ is $e^{-f(x)}$. Note that, if $f$ is positive, then $\overline{\mathcal{O}}(f)$ is effective. If $f$ is positive and plurisubharmonic, then $\overline{\mathcal{O}}(f)$ is nef. In particular, for any $a \in \mathbb{R}$, $\overline{\mathcal{O}}(a)$ is nef if and only if $a \geqslant 0$. If $\bar{L}$ is a Hermitian line bundle on $X$, we shall use the notation $\bar{L}(f)$ to denote $\bar{L} \otimes \overline{\mathcal{O}}(f)$.

We say that a Hermitian line bundle $\bar{L}$ is big if its arithmetic volume $\widehat{\operatorname{vol}}(\bar{L})$ is strictly positive. By [21, [26], $\bar{L}$ is big if and only if a positive tensor power of $\bar{L}$ can be written as the tensor product of an ample Hermitian line bundle with an effective one. Furthermore, the analogue of Fujita's approximation holds for big Hermitian line bundles, cf. [12, 27.

The arithmetic volume function $\widehat{\mathrm{vol}}$ is actually a limit (cf. [11]): one has

$$
\widehat{\operatorname{vol}}(L)=\lim _{n \rightarrow \infty} \frac{\widehat{h}^{0}\left(X, \bar{L}^{\otimes n}\right)}{n^{d+1} /(d+1) !} .
$$

Moreover, it is a birational invariant which is continuous on $\widehat{\operatorname{Pic}}(X)_{\mathbb{Q}}$, and can be continuously extended to $\widehat{\operatorname{Pic}}(X)_{\mathbb{R}}$, cf. [21, 22]. The analogue of Siu's inequality and the log-concavity hold for vol, cf. [26, $2 \mathbf{2 7}]$.

Remark 2.1. - 1) In [28] and [20], the notions of ample or nef line bundles were reserved for line bundles with smooth metrics, which is not the case here.

2) Note that there exists another (non-equivalent) definition of arithmetic volume function in the literature. See $[\mathbf{3}, \S 10.1]$ and $[\mathbf{9}, \S 5]$ where the "arithmetic volume" of a Hermitian line bundle $\bar{L}$ was defined as the following number:

$$
S(\bar{L}):=\lim _{n \rightarrow+\infty} \frac{\chi\left(\pi_{*}\left(\bar{L}^{\otimes n}\right)\right)}{n^{d+1} /(d+1) !} \in[-\infty,+\infty[,
$$

which is also called sectional capacity in the terminology of [25]. However, in the analogy between Arakelov geometry and relative algebraic geometry over a regular curve, it is (1) that corresponds to the geometric volume function. Note that one always has

$$
\widehat{\operatorname{vol}}(\bar{L}) \geqslant \lim _{n \rightarrow+\infty} \frac{\chi\left(\pi_{*}\left(\bar{L}^{\otimes n}\right)\right)}{n^{d+1} /(d+1) !},
$$

and the equality holds when $\bar{L}$ is nef. Under this assumption, both quantities are equal to the intersection number $\widehat{c}_{1}(\bar{L})^{d+1}$. This is a consequence of the HilbertSamuel formula. See [16, 1, 28, 2, 24, 21] for details.

In the following, we present some properties of nef line bundles. Note that Propositions 2.2 and 2.3 have been proved in [20, §2] for Hermitian line bundles with smooth metrics. Here we adapt these results to continuous metric case by using the continuity of intersection numbers.

Proposition 2.2. - Let $\bar{N}$ be a Hermitian line bundle on $X$ which is vertically nef. Assume that for any $x \in X(\bar{K})$, one has $h_{\bar{N}}(x) \geqslant 0$, then the Hermitian line bundle $\bar{N}$ is nef. 
Proof. - Choose an ample Hermitian line bundle $\bar{A}$ on $X$ such that $h_{\bar{A}}$ has strictly positive lower bound. For any integer $n \geqslant 1$, let $\bar{L}_{n}:=\left(L_{n},\left(\|\cdot\|_{\sigma}\right)_{\sigma: K \rightarrow \mathbb{C}}\right)$ be the tensor product $\bar{N}^{\otimes n} \otimes \bar{A}$. The height function $h_{\bar{L}_{n}}$ is bounded from below by a strictly positive number $\varepsilon_{n}$. Note that the metrics of $\bar{L}_{n}$ are semi-positive. By [19, Theorem 4.6.1] (see also [24, §3.9]), there exists a sequence of smooth positive metric families $\left(\alpha_{m}\right)_{m \geqslant 1}$ with $\alpha_{m}=\left(\|\cdot\|_{\sigma, m}\right)_{\sigma: K \rightarrow \mathbb{C}}$, such that $\|\cdot\|_{\sigma, m}$ converges uniformly to $\|\cdot\|_{\sigma}$ when $m$ tends to the infinity. Denote by $\bar{L}_{n, m}=\left(L_{n}, \alpha_{m}\right)$. For sufficiently large $m, h_{\bar{L}_{n, m}}$ is bounded from below by $\varepsilon_{n} / 2$. Thus [28, Corollary 5.7] implies that, for any integer subscheme $Y$ of $X$ which is flat over $\operatorname{Spec} \mathcal{O}_{K}$, one has $n^{-\operatorname{dim} Y} \widehat{c}_{1}\left(\left.\bar{L}_{n, m}\right|_{Y}\right) \operatorname{dim} Y \geqslant 0$. By passing successively $m$ and $n$ to the infinity, one obtains $\widehat{c}_{1}\left(\left.\bar{N}\right|_{Y}\right)^{\operatorname{dim} Y} \geqslant 0$. Therefore $\bar{N}$ is nef.

We say that a Hermitian line bundle $\bar{L}$ on $X$ is integrable if there exist two ample Hermitian line bundles $\bar{A}_{1}$ and $\bar{A}_{2}$ such that $\bar{L}=\bar{A}_{1} \otimes \bar{A}_{2}^{\vee}$. Denote by $\widehat{\operatorname{Int}}(X)$ the subgroup of $\widehat{\operatorname{Pic}}(X)$ formed by all integrable Hermitian line bundles. If $\left(\bar{L}_{i}\right)_{i=0}^{d}$ is a family of integrable Hermitian line bundles on $X$, then the intersection number

$$
\widehat{c}_{1}\left(\bar{L}_{0}\right) \cdots \widehat{c}_{1}\left(\bar{L}_{d}\right)
$$

is defined (see [28, Lemma 6.5], [29, §1] and [19] §5). Furthermore, it is a symmetric multi-linear form which is continuous in each $\bar{L}_{i}$. Namely, for any family $\left(\bar{M}_{i}\right)_{i=0}^{d}$ of integrable Hermitian line bundles, one has

$$
\lim _{n \rightarrow+\infty} n^{-d-1} \widehat{c}_{1}\left(\bar{L}_{0}^{\otimes n} \otimes \bar{M}_{0}\right) \cdots \widehat{c}_{1}\left(\bar{L}_{d}^{\otimes n} \otimes \bar{M}_{d}\right)=\widehat{c}_{1}\left(\bar{L}_{0}\right) \cdots \widehat{c}_{1}\left(\bar{L}_{d}\right) .
$$

Proposition 2.3. - Let $\left(\bar{L}_{i}\right)_{i=0}^{d-1}$ be a family of nef Hermitian line bundles on $X$ and $\bar{M}$ be an integrable Hermitian line bundle on $X$ which is effective. Then

$$
\widehat{c}_{1}\left(\bar{L}_{0}\right) \cdots \widehat{c}_{1}\left(\bar{L}_{d-1}\right) \widehat{c}_{1}(\bar{M}) \geqslant 0
$$

Proof. - Choose an ample Hermitian line bundle $\bar{A}$ on $X$ such that $h_{\bar{A}}$ is bounded from below by some strictly positive number. By virtue of the proof of Proposition 2.2. for any $i \in\{0, \cdots, d-1\}$ and any integer $n \geqslant 1$, there exists a sequence of nef Hermitian line bundles with smooth metrics $\left(\bar{L}_{i, n}^{(m)}\right)_{m \geqslant 1}$ whose underlying line bundle is $L_{i}^{\otimes n} \otimes A$ and whose metrics converge uniformly to that of $\bar{L}_{i}^{\otimes n} \otimes \bar{A}$. By [20, Proposition 2.3], one has

$$
\widehat{c}_{1}\left(\bar{L}_{0}^{\otimes n} \otimes \bar{A}\right) \cdots \widehat{c}_{1}\left(\bar{L}_{d-1}^{\otimes n} \otimes \bar{A}\right) \widehat{c}_{1}(\bar{M}) \geqslant 0 .
$$

By passing to limit, one obtains (3).

Remark 2.4. - Using the same method, we can prove that, if $\left(\bar{L}_{i}\right)_{i=0}^{d}$ is a family of nef Hermitian line bundles on $X$, then

$$
\widehat{c}_{1}\left(\bar{L}_{0}\right) \cdots \widehat{c}_{1}\left(\bar{L}_{d}\right) \geqslant 0 .
$$

Proposition 2.5. - Let $\bar{L}$ be a Hermitian line bundle on $X$ such that $c_{1}(\bar{L})$ is semipositive in the sense of current on $X(\mathbb{C})$. Assume that there exists an integer $n>0$ 
HUAYI CHEN

such that $L^{\otimes n}$ is generated by its effective sections. Then the Hermitian line bundle $\bar{L}$ is nef.

Proof. - Since $L^{\otimes n}$ is generated by its sections, the line bundle $L$ is nef relatively to $\pi$. After Proposition 2.2, it suffices to verify that, for any $x \in X(\bar{K})$, one has $h_{\bar{L}}(x) \geqslant 0$. For any integer $m \geqslant 1$, let $B_{m}=\pi_{*}\left(L^{\otimes m}\right)$ and let $B_{m}^{[0]}$ be the saturated sub- $\mathcal{O}_{K}$-module of $B_{m}$ generated by effective sections. Since $L^{\otimes n}$ is generated by its effective sections, also is $L^{\otimes n p}$ for any integer $p \geqslant 1$. In particular, one has surjective homomorphisms $x^{*} \pi^{*} B_{p n, K_{x}}^{[0]} \rightarrow x^{*} L_{K_{x}}^{\otimes n p}$. By slope inequality (see [5. Appendix A]), one has $n p h_{\bar{L}}(x) \geqslant \widehat{\mu}_{\min }\left(\bar{B}_{n p}^{[0]}\right)$. By passing to limit, one obtains $h_{\bar{L}}(x) \geqslant 0$.

We say that a Hermitian line bundle $\bar{L}$ on $X$ is free if $c_{1}(\bar{L})$ is semi-positive in the sense of current on $X(\mathbb{C})$ and if some positive tensor power of $L$ is generated by effective global sections. We denote by $\widehat{\operatorname{Fr}}(X)$ the subgroup of $\widehat{\operatorname{Pic}}(X)$ consisting of free Hermitian line bundles. After Proposition 2.5, one has $\widehat{\operatorname{Fr}}(X) \subset \widehat{\operatorname{Nef}}(X)$.

Unlike the ampleness, the properties of being big, nef, or free are all invariant by birational modifications. That is, if $\nu: X^{\prime} \rightarrow X$ is a birational projective morphism, and if $\bar{L}$ is a Hermitian line bundle on $X$ which is big (resp. nef, free), then also is $\nu^{*}(\bar{L})$.

\section{Positive intersection product}

In this section, we shall define the positive intersection product for big (nonnecessarily integral) Hermitian line bundles. When all Hermitian line bundles are nef, the positive intersection product coincides with the usual intersection product. Furthermore, the highest positive auto-intersection number is just the arithmetic volume of the Hermitian line bundle. We shall use the positive intersection product to interpret the differential of the arithmetic volume function.

\subsection{Admissible decompositions. -}

Definition 3.1. - Let $\bar{L}$ be a big Hermitian line bundle on $X$. We call admissible decomposition of $\bar{L}$ any triplet $(\nu, \bar{N}, p)$, where

1) $\nu: X^{\prime} \rightarrow X$ is a birational projective morphism,

2) $\bar{N}$ is a free Hermitian line bundle on $X^{\prime}$,

3) $p \geqslant 1$ is an integer such that $\nu^{*}\left(\bar{L}^{\otimes p}\right) \otimes \bar{N}^{\vee}$ is effective.

Denote by $\Theta(\bar{L})$ the set of all admissible decompositions of $\bar{L}$.

We introduce an order relation on the set $\Theta(\bar{L})$. Let $D_{i}=\left(\nu_{i}: X_{i} \rightarrow X, \bar{N}_{i}, p_{i}\right)$ $(i=1,2)$ be two admissible decompositions of $\bar{L}$. We say that $D_{1}$ is superior to $D_{2}$ and we denote by $D_{1} \succ D_{2}$ if $p_{2}$ divides $p_{1}$ and if there exists a projective birational morphism $\eta: X_{1} \rightarrow X_{2}$ such that $\nu_{2} \eta=\nu_{1}$ and that $\bar{N}_{1} \otimes\left(\eta^{*} \bar{N}_{2}\right)^{\vee \otimes\left(p_{1} / p_{2}\right)}$ is effective.

Remark 3.2. - (1) Assume that $D=\left(\nu: X^{\prime} \rightarrow X, \bar{N}, p\right)$ is an admissible decomposition of $\bar{L}$. Then for any birational projective morphism $\eta: X^{\prime \prime} \rightarrow X^{\prime}$, the 
triplet $\eta^{*} D:=\left(\nu \eta, \eta^{*} \bar{N}, p\right)$ is also an admissible decomposition of $\bar{L}$, and one has $\eta^{*} D \succ D$.

(2) Assume that $D=(\nu, \bar{N}, p)$ is an admissible decomposition of $\bar{L}$. Then for any integer $n \geqslant 1, D_{n}=\left(\nu, \bar{N}^{\otimes n}, n p\right)$ is also an admissible decomposition of $\bar{L}$. Furthermore, one has $D_{n} \succ D$.

(3) Assume that $D_{1}=\left(\nu, \bar{N}_{1}, p\right)$ and $D_{2}=\left(\nu, \bar{N}_{2}, q\right)$ are two admissible decompositions of $\bar{L}$ whose underlying birational projective morphisms are the same. Then $D_{1} \otimes D_{2}:=\left(\nu, \bar{N}_{1} \otimes \bar{N}_{2}, p+q\right)$ is an admissible decomposition of $\bar{L}$.

(4) Assume that $\bar{M}$ is an effective Hermitian line bundle on $X$. By definition, any admissible decomposition of $\bar{L}$ is also an admissible decomposition of $\bar{L} \otimes \bar{M}$.

In the following proposition, we show that the set $\Theta(\bar{L})$ is filtered with respect to the order $\succ$.

Proposition 3.3. - if $D_{1}$ and $D_{2}$ are two admissible decompositions of $\bar{L}$, then there exists an admissible decomposition $D$ of $\bar{L}$ such that $D \succ D_{1}$ and $D \succ D_{2}$.

Proof. - After Remark 3.2 (1)(2), we may assume that the first and the third components of $D_{1}$ and $D_{2}$ are the same. Assume that $D_{1}=\left(\nu, \bar{N}_{1}, p\right)$ and $D_{2}=$ $\left(\nu, \bar{N}_{2}, p\right)$, where $\nu: X^{\prime} \rightarrow X$ is a birational projective morphism. Let $\bar{M}_{i}=\nu^{*} \bar{L}^{\otimes p} \otimes$ $\bar{N}_{i}^{\vee}(i=1,2)$. Since $\bar{M}_{1}$ and $\bar{M}_{2}$ are effective, there exist homomorphisms $u_{i}: M_{i}^{\vee} \rightarrow$ $\mathcal{O}_{X^{\prime}}$ corresponding to effective sections $s_{i}: \mathcal{O}_{X^{\prime}} \rightarrow M_{i}(i=1,2)$. Let $\eta: X^{\prime \prime} \rightarrow X^{\prime}$ be the blow up of the ideal sheaf $\operatorname{Im}\left(u_{1} \oplus u_{2}\right)$. Let $M$ be the exceptional line bundle and $s: \mathcal{O}_{X^{\prime \prime}} \rightarrow M$ be the section which trivializes $M$ outside the exceptional divisor. The canonical surjective homomorphism $\eta^{*}\left(M_{1}^{\vee} \oplus M_{2}^{\vee}\right) \rightarrow M^{\vee}$ induces by duality an injective homomorphism $\varphi: M \rightarrow M_{1} \oplus M_{2}$. We equip $M_{1} \oplus M_{2}$ with metrics $\left(\|\cdot\|_{\sigma}\right)_{\sigma: K \rightarrow \mathbb{C}}$ such that, for any $x \in X_{\sigma}^{\prime \prime}(\mathbb{C})$ and any section $(u, v)$ of $M_{1, \sigma} \oplus M_{2, \sigma}$ over a neighbourhood of $x$, one has $\|(u, v)\|_{\sigma}(x)=\max \left\{\|u\|_{\sigma, 1}(x),\|v\|_{\sigma, 2}(x)\right\}$. As $\varphi s=\left(\eta^{*} s_{1}, \eta^{*} s_{2}\right)$, and the sections $s_{1}$ and $s_{2}$ are effective, one obtains that the section $s$ is also effective. Let $\bar{N}=(\nu \eta)^{*} \bar{L}^{\otimes p} \otimes \bar{M}^{\vee}$. One has a natural surjective homomorphism

$$
\psi: \eta^{*} N_{1} \oplus \eta^{*} N_{2} \longrightarrow N .
$$

Furthermore, if we equip $\eta^{*} N_{1} \oplus \eta^{*} N_{2}$ with metrics $\left(\|\cdot\|_{\sigma}\right)_{\sigma: K \rightarrow \mathbb{C}}$ such that, for any $x \in X_{\sigma}^{\prime \prime}(x),\|(u, v)\|_{\sigma}(x)=\|u\|_{\sigma}(x)+\|v\|_{\sigma}(x)$, then the metrics on $N$ are just the quotient metrics by the surjective homomorphism $\psi$, which are semi-positive since the metrics of $\eta^{*} \bar{N}_{1}$ and of $\eta^{*} \bar{N}_{2}$ are. As both Hermitian line bundles $N_{1}$ and $N_{2}$ are generated by effective global sections, also is $\bar{N}$. Therefore, $(\nu \eta, \bar{N}, p)$ is an admissible decomposition of $\bar{L}$, which is superior to both $D_{1}$ and $D_{2}$.

3.2. Intersection of admissible decompositions. - Let $\left(\bar{L}_{i}\right)_{i=0}^{d}$ be a family of Hermitian line bundles on $X$. Let $m \in\{0, \cdots d\}$. Assume that $\bar{L}_{i}$ is big for any $i \in\{0, \cdots, m\}$ and is integrable for any $i \in\{m+1, \cdots, d\}$. For any $i \in\{0, \cdots, m\}$, let $D_{i}=\left(\nu_{i}: X_{i} \rightarrow X, \bar{N}_{i}, p_{i}\right)$ be an admissible decomposition of $\bar{L}_{i}$. Choose a birational projective morphism $\nu: X^{\prime} \rightarrow X$ which factorizes through $\nu_{i}$ for each $i \in\{0, \cdots, m\}$. Denote by $\eta_{i}: X_{i} \rightarrow X$ the projective birational morphism such that 
$\nu=\nu_{i} \eta_{i}(0 \leqslant i \leqslant m)$. Define $\left(D_{0} \cdots D_{m}\right) \cdot \widehat{c}_{1}\left(\bar{L}_{m+1}\right) \cdots \widehat{c}_{1}\left(\bar{L}_{d}\right)$ as the normalized intersection product

$$
\widehat{c}_{1}\left(\eta_{0}^{*} \bar{N}_{0}\right) \cdots \widehat{c}_{1}\left(\eta_{m}^{*} \bar{N}_{m}\right) \widehat{c}_{1}\left(\nu^{*} \bar{L}_{m+1}\right) \cdots \widehat{c}_{1}\left(\nu^{*} \bar{L}_{d}\right) \prod_{i=0}^{m} p_{i}^{-1} .
$$

This definition does not depend on the choice of $\nu$.

Proposition 3.4. - Let $\left(\bar{L}_{i}\right)_{0 \leqslant i \leqslant d}$ be a family of Hermitian line bundles on $X$. Let $m \in\{0, \cdots, d\}$. Assume that $\bar{L}_{i}$ is big for $i \in\{0, \cdots, m\}$, and is nef for $i \in\{m+1, \cdots, d\}$. For any $i \in\{0, \cdots, m\}$, let $D_{i}$ and $D_{i}^{\prime}$ be two admissible decompositions of $\bar{L}_{i}$ such that $D_{i} \succ D_{i}^{\prime}$. Then

$$
\left(D_{0} \cdots D_{m}\right) \cdot \widehat{c}_{1}\left(\bar{L}_{m+1}\right) \cdots \widehat{c}_{1}\left(\bar{L}_{d}\right) \geqslant\left(D_{0}^{\prime} \cdots D_{m}^{\prime}\right) \cdot \widehat{c}_{1}\left(\bar{L}_{m+1}\right) \cdots \widehat{c}_{1}\left(\bar{L}_{d}\right)
$$

Proof. - By substituting progressively $D_{i}$ by $D_{i}^{\prime}$, it suffices to prove that

$$
\left(D_{0} \cdot D_{1} \cdots D_{m}\right) \cdot \widehat{c}_{1}\left(\bar{L}_{m+1}\right) \cdots \widehat{c}_{1}\left(\bar{L}_{d}\right) \geqslant\left(D_{0}^{\prime} \cdot D_{1} \cdots D_{m}\right) \cdot \widehat{c}_{1}\left(\bar{L}_{m+1}\right) \cdots \widehat{c}_{1}\left(\bar{L}_{d}\right),
$$

which is a consequence of Proposition 2.3.

Corollary 3.5. - With the notation and the assumptions of Proposition 3.4, the supremum

$$
\sup \left\{\left(D_{0} \cdots D_{m}\right) \cdot \widehat{c}_{1}\left(\bar{L}_{m+1}\right) \cdots \widehat{c}_{1}\left(\bar{L}_{d}\right) \mid 0 \leqslant i \leqslant m, D_{i} \in \Theta\left(\bar{L}_{i}\right)\right\}
$$

exists in $\mathbb{R}_{\geqslant 0}$.

Proof. - For any $i \in\{0, \cdots, m\}$, let $\bar{A}_{i}$ be an arithmetically ample Hermitian line bundle on $X$ such that $\bar{A}_{i} \otimes \bar{L}_{i}^{\vee}$ is effective. Then all numbers of the set (6) is bounded from above by $\widehat{c}_{1}\left(\bar{A}_{0}\right) \cdots \widehat{c}_{1}\left(\bar{A}_{m}\right) \cdot \widehat{c}_{1}\left(\bar{L}_{m+1}\right) \cdots \widehat{c}_{1}\left(\bar{L}_{d}\right)$.

3.3. Positive intersection product. - Let $\left(\bar{L}_{i}\right)_{i=0}^{m}$ be a family of big Hermitian line bundles on $X$, where $0 \leqslant m \leqslant d$. Denote by $\left\langle\widehat{c}_{1}\left(\bar{L}_{0}\right) \cdots \widehat{c}_{1}\left(\bar{L}_{m}\right)\right\rangle$ the function on $\widehat{\operatorname{Nef}}(X)^{d-m}$ which sends a family of nef Hermitian line bundles $\left(\bar{L}_{j}\right)_{j=m+1}^{d}$ to the supremum

$$
\sup \left\{\left(D_{0} \cdots D_{m}\right) \cdot \widehat{c}_{1}\left(\bar{L}_{m+1}\right) \cdots \widehat{c}_{1}\left(\bar{L}_{d}\right) \mid 0 \leqslant i \leqslant m, D_{i} \in \Theta\left(\bar{L}_{i}\right)\right\} .
$$

Since all $\Theta\left(\bar{L}_{i}\right)$ are filtered, this function is additive in each $\bar{L}_{j}(m+1 \leqslant j \leqslant d)$. Thus it extends naturally to a multi-linear function on $\widehat{\operatorname{nnt}}(X)$ which we still denote by $\left\langle\widehat{c}_{1}\left(\bar{L}_{0}\right) \cdots \widehat{c}_{1}\left(\bar{L}_{m}\right)\right\rangle$, called the positive intersection product of $\left(\bar{L}_{i}\right)_{i=0}^{m}$.

Remark 3.6. - (1) If all Hermitian vector bundles $\left(\bar{L}_{i}\right)_{i=0}^{m}$ are nef, then the positive intersection product coincides with the usual intersection product.

(2) The positive intersection product is homogeneous in each $\bar{L}_{i}(0 \leqslant i \leqslant m)$. However, in general it is not additive in each variable. If we consider it as a function on $\widehat{\operatorname{Nef}}(X)$, then it is super-additive in each variable. 
(3) Assume that all Hermitian line bundles $\left(\bar{L}_{i}\right)_{i=0}^{m}$ are the same. That is, $\bar{L}_{0}=\cdots=$ $\bar{L}_{m}=\bar{L}$. We use the expression $\left\langle\widehat{c}_{1}(\bar{L})^{m+1}\right\rangle$ to denote the positive intersection product

$$
\langle\underbrace{\widehat{c}_{1}(\bar{L}) \cdots \widehat{c}_{1}(\bar{L})}_{m+1 \text { copies }}\rangle \text {. }
$$

With this notation, for any $\left(\bar{L}_{j}\right)_{j=m+1}^{d} \in \widehat{\operatorname{Nef}}(X)^{d-m}$, one has

$$
\left\langle\widehat{c}_{1}(\bar{L})^{m}\right\rangle \cdot \widehat{c}_{1}\left(\bar{L}_{m+1}\right) \cdots \widehat{c}_{1}\left(\bar{L}_{d}\right)=\sup _{D \in \Theta(\bar{L})}(D \cdots D) \cdot \widehat{c}_{1}\left(\bar{L}_{m+1}\right) \cdots \widehat{c}_{1}\left(\bar{L}_{d}\right) .
$$

This equality comes from the fact that the ordered set $\Theta(\bar{L})$ is filtered (Proposition 3.3) and from the comparison (5). In particular, the Fujita's approximation theorem (see [12] and [27]) implies that $\left\langle\widehat{c}_{1}(\bar{L})^{d+1}\right\rangle=\widehat{\operatorname{vol}}(\bar{L})$.

Lemma 3.7. - Let $\left(\bar{L}_{i}\right)_{i=0}^{m}$ be a family of big Hermitian line bundles on $X$, where $m \in\{0, \cdots, d\}$. For any $i \in\{0, \cdots, m\}$, let $\bar{M}_{i}$ be an effective Hermitian line bundle on $X$ and let $\bar{N}_{i}=\bar{L}_{i} \otimes \bar{M}_{i}$. Then one has

$$
\left\langle\widehat{c}_{1}\left(\bar{L}_{0}\right) \cdots \widehat{c}_{1}\left(\bar{L}_{m}\right)\right\rangle \geqslant\left\langle\widehat{c}_{1}\left(\bar{N}_{0}\right) \cdots \widehat{c}_{1}\left(\bar{N}_{m}\right)\right\rangle \text {, }
$$

where we have considered the positive intersection products as functions on $\widehat{\operatorname{Nef}}(X)^{d-m}$.

Proof. - By Remark 3.6 (4), if $D_{i}$ is an admissible decomposition of $\bar{L}_{i}$, then it is also an admissible decomposition of $\bar{L}_{i}$. Hence by the definition of the positive intersection product, the inequality (7) is true.

The following proposition shows that the positive intersection product is continuous in each variable.

Proposition 3.8. - Let $\left(\bar{L}_{i}\right)_{0 \leqslant i \leqslant m}$ be a family of big Hermitian line bundles on $X$, where $m \in\{0, \cdots, d\}$. Let $\left(\bar{M}_{i}\right)_{0 \leqslant i \leqslant m}$ be a family of Hermitian line bundles on $\mathscr{X}$. Then

$$
\lim _{n \rightarrow \infty} n^{-m}\left\langle\widehat{c}_{1}\left(\bar{L}_{0}^{\otimes n} \otimes \bar{M}_{0}\right) \cdots \widehat{c}_{1}\left(\bar{L}_{m}^{\otimes n} \otimes \bar{M}_{m}\right)\right\rangle=\left\langle\widehat{c}_{1}\left(\bar{L}_{0}\right) \cdots \widehat{c}_{1}\left(\bar{L}_{m}\right)\right\rangle
$$

Proof. - We consider firstly both positive intersection products as functions on $\widehat{\operatorname{Nef}}(X)$. Let $\alpha_{n}=\left\langle\widehat{c}_{1}\left(\bar{L}_{0}^{\otimes n} \otimes \bar{M}_{0}\right) \cdots \widehat{c}_{1}\left(\bar{L}_{m}^{\otimes n} \otimes \bar{M}_{m}\right)\right\rangle$. Since $\bar{L}_{i}$ is big, there exists an integer $q \geqslant 1$ such that the Hermitian line bundles $\bar{L}_{i}^{\otimes q} \otimes \bar{M}_{i}$ and $\bar{L}_{i}^{\otimes q} \otimes \bar{M}_{i}^{\vee}$ are both effective. Thus the Lemma 3.7 implies that

$$
\begin{aligned}
& \alpha_{n} \geqslant\left\langle\widehat{c}_{1}\left(\bar{L}_{0}^{\otimes(n-q)}\right) \cdots \widehat{c}_{1}\left(\bar{L}_{m}^{\otimes(n-q)}\right)\right\rangle=(n-q)^{m}\left\langle\widehat{c}_{1}\left(\bar{L}_{0}\right) \cdots \widehat{c}_{1}\left(\bar{L}_{m}\right)\right\rangle, \\
& \alpha_{n} \leqslant\left\langle\widehat{c}_{1}\left(\bar{L}_{0}^{\otimes(n+q)}\right) \cdots \widehat{c}_{1}\left(\bar{L}_{m}^{\otimes(n+q)}\right)\right\rangle=(n+q)^{m}\left\langle\widehat{c}_{1}\left(\bar{L}_{0}\right) \cdots \widehat{c}_{1}\left(\bar{L}_{m}\right)\right\rangle .
\end{aligned}
$$

By passing to limit, we obtain (8) as an equality of functions on $\widehat{\operatorname{Nef}}(X)^{d-m}$. The general case follows from the multi-linearity. 
Remark 3.9. - Proposition 3.8 implies in particular that, if $\left(f_{n}^{(i)}\right)_{n \geqslant 1}(i=$ $0,1, \cdots, m)$ are families of continuous functions on $X(\mathbb{C})$ which converge uniformly to zero. Then one has

$$
\lim _{n \rightarrow+\infty}\left\langle\widehat{c}_{1}\left(\bar{L}_{0}\left(f_{0}\right)\right) \cdots \widehat{c}_{m}\left(\bar{L}_{m}\left(f_{m}\right)\right)\right\rangle=\left\langle\widehat{c}_{1}\left(\bar{L}_{0}\right) \cdots \widehat{c}_{m}\left(\bar{L}_{m}\right)\right\rangle .
$$

In particular, the mapping

$$
t \mapsto\left\langle\widehat{c}_{1}\left(\bar{L}_{0}(t)\right) \cdots \widehat{c}_{1}\left(\bar{L}_{m}(t)\right)\right\rangle
$$

is continuous on the (open) interval that it it well defined.

Proposition 3.10. - Let $\left(\bar{L}_{i}\right)_{i=0}^{d-1}$ be a family of big Hermitian line bundles on X. If $\bar{M}$ is an effective integrable Hermitian line bundle on $X$, then

$$
\left\langle\widehat{c}_{1}\left(\bar{L}_{0}\right) \cdots \widehat{c}_{1}\left(\bar{L}_{d-1}\right)\right\rangle \cdot \widehat{c}_{1}(\bar{M}) \geqslant 0 .
$$

Proof. - This is a direct consequence of Proposition 2.3

Remark 3.11. - Proposition 3.10 permits us to extend the function $\left\langle\widehat{c}_{1}\left(\bar{L}_{0}\right) \cdots \widehat{c}_{1}\left(\bar{L}_{d-1}\right)\right\rangle$ on $\widehat{\operatorname{Pic}}(X)$. Let $\bar{M}$ be an arbitrary Hermitian line bundle on $X$. By Weierstrass-Stone theorem, there exists a sequence $\left(f_{n}\right)_{n \geqslant 1}$ of continuous functions on $X(\mathbb{C})$ which converges uniformly to 0 , and such that $\bar{M}\left(f_{n}\right)$ is of smooth metrics for any $n$. Thus $\bar{M}\left(f_{n}\right)$ is integrable and $a_{n}=\left\langle\widehat{c}_{1}\left(\bar{L}_{0}\right) \cdots \widehat{c}_{1}\left(\bar{L}_{d-1}\right)\right\rangle \cdot \widehat{c}_{1}\left(\bar{M}\left(f_{n}\right)\right)$ is well defined. Let $\varepsilon_{n, m}=\left\|f_{n}-f_{m}\right\|_{\text {sup }}$. Choose an ample Hermitian line bundle $\bar{A}$ such that $\bar{A} \otimes \bar{L}_{i}^{\vee}$ is effective for any $i \in\{0, \cdots, d-1\}$. Note that

$$
\begin{aligned}
a_{n}-a_{m} & =\left\langle\widehat{c}_{1}\left(\bar{L}_{0}\right) \cdots \widehat{c}_{1}\left(\bar{L}_{d-1}\right)\right\rangle \cdot \widehat{c}_{1}\left(\overline{\mathcal{O}}\left(f_{n}-f_{m}\right)\right) \\
& \leqslant\left\langle\widehat{c}_{1}\left(\bar{L}_{0}\right) \cdots \widehat{c}_{1}\left(\bar{L}_{d-1}\right)\right\rangle \cdot \widehat{c}_{1}\left(\overline{\mathcal{O}}\left(\varepsilon_{n, m}\right)\right) \\
& \leqslant\left\langle\widehat{c}_{1}(\bar{A})^{d}\right\rangle \cdot \widehat{c}_{1}\left(\overline{\mathcal{O}}\left(\varepsilon_{n, m}\right)\right)=\varepsilon_{n, m} c_{1}\left(A_{K}\right)^{d}
\end{aligned}
$$

By interchanging the roles of $n$ and $m$ in (9) and then combining the two inequalities, one obtains $\left|a_{n}-a_{m}\right| \leqslant \varepsilon_{n, m} c_{1}\left(A_{K}\right)^{d}$. Therefore, $\left(a_{n}\right)_{n \geqslant 1}$ is a Cauchy sequence which converges to a real number which we denote by $\left\langle\widehat{c}_{1}\left(\bar{L}_{0}\right) \cdots \widehat{c}_{1}\left(\bar{L}_{d-1}\right)\right\rangle \cdot \widehat{c}_{1}(\bar{M})$. By an argument similar to the inequality (9), this definition does not depend on the choice of the sequence $\left(f_{n}\right)_{n \geqslant 1}$. The extended function is additive on $\widehat{\operatorname{Pic}}(X)$, which is positive on the subgroup of effective Hermitian line bundles, and satisfies the conclusion of Proposition 3.8 .

\section{Differentiability of the arithmetic volume function}

In this section, we establish the differentiability of the arithmetic volume function. We begin by presenting the following lemma, which is analogous to [8, Corollary 3.4].

Lemma 4.1. - Let $\bar{L}$ and $\bar{N}$ be two nef Hermitian line bundles on $X$. Let $\bar{M}$ be an integrable Hermitian line bundle on $X$. Assume that $\bar{M} \otimes \bar{N}$ and $\bar{M}^{\vee} \otimes \bar{N}$ are nef 
and that $\bar{L}^{\vee} \otimes \bar{N}$ is effective. Then there exists a constant $C>0$ only depending on $d$ such that

$$
\widehat{\operatorname{vol}}\left(\bar{L}^{\otimes n} \otimes \bar{M}\right) \geqslant n^{d+1} \widehat{\operatorname{vol}}(\bar{L})+(d+1) n^{d} \widehat{c}_{1}(\bar{L})^{d} \widehat{c}_{1}(\bar{M})-C \widehat{\operatorname{vol}}(\bar{N}) n^{d-1} .
$$

Proof. - Recall that (see [26. Theorem 2.2], see also [21, Theorem 5.6]) if $\bar{A}$ and $\bar{B}$ are two nef Hermitian line bundles on $X$, then

$$
\widehat{\operatorname{vol}}\left(\bar{B} \otimes \bar{A}^{\vee}\right) \geqslant \widehat{c}_{1}(\bar{B})^{d+1}-\widehat{c}_{1}(\bar{B})^{d} \widehat{c}_{1}(\bar{A}) .
$$

Let $\bar{B}=\bar{L}^{\otimes n} \otimes \bar{M} \otimes \bar{N}$. It is a nef Hermitian line bundle on $X$. If one applies (10) on $\bar{B}$ and on $\bar{A}=\bar{N}$, one obtains

$$
\begin{aligned}
\widehat{\operatorname{vol}}\left(\bar{L}^{\otimes n} \otimes \bar{M}\right) & =\widehat{\operatorname{vol}}\left(\bar{B} \otimes \bar{N}^{\vee}\right) \geqslant \widehat{c}_{1}(\bar{B})^{d+1}-(d+1) \widehat{c}_{1}(\bar{B})^{d} \widehat{c}_{1}(\bar{N}) \\
& =n^{d+1} \widehat{c}_{1}(\bar{L})^{d+1}+(d+1) n^{d} \widehat{c}_{1}(\bar{L})^{d} \widehat{c}_{1}(\bar{M})+O\left(n^{d-1}\right),
\end{aligned}
$$

where the implicit constant is a linear combination of intersection numbers of Hermitian line bundles of the form $\bar{L}$ or $\bar{M} \otimes \bar{N}$, and hence can be bounded from above by a multiple of $\widehat{c}_{1}(\bar{N})^{d+1}=\widehat{\operatorname{vol}}(\bar{N})$, according to Proposition 2.3.

Remark 4.2. - In [26], Yuan has actually proved a stronger inequality by replacing the $\widehat{\operatorname{vol}}\left(\bar{B} \otimes \bar{A}^{\vee}\right)$ in (10) by

$$
\lim _{n \rightarrow+\infty} \frac{\chi\left(\pi_{*}\left(\bar{B}^{\otimes n} \otimes \bar{A}^{\vee \otimes n}\right)\right)}{n^{d+1} /(d+1) !} .
$$

In fact, this quantity is always bounded from above by $\widehat{\operatorname{vol}}\left(\bar{B} \otimes \bar{A}^{\vee}\right)$.

Proof of Theorem 1.1 - We first assume that the metrics of $\bar{M}$ are smooth. Choose an ample Hermitian line bundle $\bar{N}$ such that $\bar{N} \otimes \bar{M}$ and $\bar{N} \otimes \bar{M}^{\vee}$ are ample, and that $\bar{N} \otimes \bar{L}^{\vee}$ is effective. Let $D=\left(\nu: X^{\prime} \rightarrow X, \bar{A}, p\right)$ be an admissible decomposition of $\bar{L}$. One has

$$
\widehat{\operatorname{vol}}\left(\bar{L}^{\otimes n} \otimes \bar{M}\right)=p^{-d-1} \widehat{\operatorname{vol}}\left(\nu^{*} \bar{L}^{\otimes n p} \otimes \nu^{*} \bar{M}^{\otimes p}\right) \geqslant p^{-d-1} \widehat{\operatorname{vol}}\left(\bar{A}^{\otimes n} \otimes \bar{M}^{\otimes p}\right) .
$$

Note that $\bar{N}^{\otimes p} \otimes \bar{M}^{\otimes p}$ and $\bar{N}^{\otimes p} \otimes \bar{M}^{\vee \otimes p}$ are nef and

$$
\bar{N}^{\otimes p} \otimes \bar{A}^{\vee}=\left(\bar{N} \otimes \bar{L}^{\vee}\right)^{\otimes p} \otimes\left(\bar{L}^{\otimes p} \otimes \bar{A}^{\vee}\right)
$$

is effective. After Lemma 4.1 one obtains

$$
\widehat{\operatorname{vol}}\left(\bar{A}^{\otimes n} \otimes \bar{M}^{\otimes p}\right) \geqslant n^{d+1} \widehat{c}_{1}(\bar{A})^{d+1}+(d+1) n^{d} p \widehat{c}_{1}(\bar{A})^{d} \widehat{c}_{1}(\bar{M})-C p^{d+1} \widehat{\operatorname{vol}}(\bar{N}) n^{d-1} .
$$

Therefore,

$$
\widehat{\operatorname{vol}}\left(\bar{L}^{\otimes n} \otimes \bar{M}\right) \geqslant n^{d+1}\left(D^{d+1}\right)+(d+1) n^{d}\left(D^{d}\right) \cdot \widehat{c}_{1}(\bar{M})-C \widehat{\operatorname{vol}}(\bar{N}) n^{d-1} .
$$

Since $D$ is arbitrary, one has

$$
\widehat{\operatorname{vol}}\left(\bar{L}^{\otimes n} \otimes \bar{M}\right) \geqslant n^{d+1} \widehat{\operatorname{vol}}(\bar{L})+(d+1) n^{d}\left\langle\widehat{c}_{1}(\bar{L})^{d}\right\rangle \cdot \widehat{c}_{1}(\bar{M})-C \widehat{\operatorname{vol}}(\bar{N}) n^{d-1} .
$$

By passing to limit, one obtains

$$
\liminf _{n \rightarrow+\infty} \frac{\widehat{\operatorname{vol}}\left(\bar{L}^{\otimes n} \otimes \bar{M}\right)-\widehat{\operatorname{vol}}\left(\bar{L}^{\otimes n}\right)}{n^{d}} \geqslant(d+1)\left\langle\widehat{c}_{1}(\bar{L})^{d}\right\rangle \cdot \widehat{c}_{1}(\bar{M}) .
$$


HUAYI CHEN

If we apply (11) in replacing $\bar{L}$ by $\bar{L}^{\otimes n} \otimes \bar{M}, \bar{M}$ by $\bar{M}^{\vee \otimes n}$ and $\bar{N}$ by $\bar{N}^{\otimes 2 n}$, we obtain $\widehat{\operatorname{vol}}\left(\bar{L}^{\otimes n^{2}}\right) \geqslant n^{d+1} \widehat{\operatorname{vol}}\left(\bar{L}^{\otimes n} \otimes \bar{M}\right)-(d+1) n^{d+1}\left\langle\widehat{c}_{1}\left(\bar{L}^{\otimes n} \otimes \bar{M}\right)^{d}\right\rangle \cdot \widehat{c}_{1}(\bar{M})-C(2 n)^{d+1} \widehat{\operatorname{vol}}(\bar{N}) n^{d-1}$, or equivalently

$$
\widehat{\operatorname{vol}}\left(\bar{L}^{\otimes n}\right) \geqslant \widehat{\operatorname{vol}}\left(\bar{L}^{\otimes n} \otimes \bar{M}\right)-(d+1)\left\langle\widehat{c}_{1}\left(\bar{L}^{\otimes n} \otimes \bar{M}\right)^{d}\right\rangle \cdot \widehat{c}_{1}(\bar{M})-2^{d+1} \widehat{C \operatorname{vol}}(\bar{N}) n^{d-1} .
$$

Thus

$$
\begin{aligned}
\limsup _{n \rightarrow \infty} \frac{\widehat{\operatorname{vol}}\left(\bar{L}^{\otimes n} \otimes \bar{M}\right)-\widehat{\operatorname{vol}}\left(\bar{L}^{\otimes n}\right)}{n^{d}} & \leqslant \lim _{n \rightarrow \infty} n^{-d}(d+1)\left\langle\widehat{c}_{1}\left(\bar{L}^{\otimes n} \otimes \bar{M}\right)^{d}\right\rangle \cdot \widehat{c}_{1}(\bar{M}) \\
& =(d+1)\left\langle\widehat{c}_{1}(\bar{L})^{d}\right\rangle \cdot \widehat{c}_{1}(\bar{M}) .
\end{aligned}
$$

Therefore, one has

$$
\lim _{n \rightarrow+\infty} \frac{\widehat{\operatorname{vol}}\left(\bar{L}^{\otimes n} \otimes \bar{M}\right)-\widehat{\operatorname{vol}}\left(\bar{L}^{\otimes n}\right)}{n^{d}}=(d+1)\left\langle\widehat{c}_{1}(\bar{L})^{d}\right\rangle \cdot \widehat{c}_{1}(\bar{M}) .
$$

For the general case, by Weierstrass-Stone theorem, for any $\varepsilon>0$, there exist two Hermitian line bundles with smooth metrics $\bar{M}_{\varepsilon, 1}=\left(M,\left(\|\cdot\|_{\sigma, \varepsilon}^{\prime}\right)_{\sigma: K \rightarrow \mathbb{C}}\right)$ and $\bar{M}_{\varepsilon, 2}=\left(M,\left(\|\cdot\|_{\sigma, \varepsilon}^{\prime \prime}\right)_{\sigma: K \rightarrow \mathbb{C}}\right)$ such that

$$
\|\cdot\|_{\sigma, \varepsilon}^{\prime} \leqslant\|\cdot\|_{\sigma} \leqslant\|\cdot\|_{\sigma, \varepsilon}^{\prime \prime}, \quad \text { and } \max _{\sigma} \sup _{x \in X_{\sigma}(\mathbb{C})}\left|\log \|\cdot\|_{\sigma, \varepsilon}^{\prime}(x)-\log \|\cdot\|_{\sigma, \varepsilon}^{\prime \prime}(x)\right| \leqslant \varepsilon,
$$

where $\|\cdot\|_{\sigma}$ is the norm of index $\sigma$ of $\bar{M}$. Note that $\bar{M}_{\varepsilon, 2} \leqslant \bar{M} \leqslant \bar{M}_{\varepsilon, 1}$. By the special case that we have proved, one has

$$
\begin{aligned}
& (d+1)\left\langle\widehat{c}_{1}(\bar{L})^{d}\right\rangle \cdot \widehat{c}_{1}\left(\bar{M}_{\varepsilon, 2}\right) \leqslant \liminf _{n \rightarrow+\infty} \frac{\widehat{\operatorname{vol}}\left(\bar{L}^{\otimes n} \otimes \bar{M}\right)-\widehat{\operatorname{vol}}\left(\bar{L}^{\otimes n}\right)}{n^{d}} \\
\leqslant & \limsup _{n \rightarrow+\infty} \frac{\widehat{\operatorname{vol}}\left(\bar{L}^{\otimes n} \otimes \bar{M}\right)-\widehat{\operatorname{vol}}\left(\bar{L}^{\otimes n}\right)}{n^{d}} \leqslant(d+1)\left\langle\widehat{c}_{1}(\bar{L})^{d}\right\rangle \cdot \widehat{c}_{1}\left(\bar{M}_{\varepsilon, 1}\right)
\end{aligned}
$$

Let $f_{\varepsilon}: X(\mathbb{C}) \rightarrow \mathbb{R}$ be the function such that $\log \|\cdot\|_{\sigma, \varepsilon}^{\prime \prime}(x)=\log \|\cdot\|_{\sigma, \varepsilon}^{\prime}(x)+f_{\varepsilon}(x)$. Denote by $\overline{\mathcal{O}}\left(f_{\varepsilon}\right)$ the Hermitian line bundle on $X$ whose underlying Hermitian line bundle is trivial, and such that $\|\mathbf{1}\|(x)=e^{-f_{\varepsilon}(x)}$. It is an effective Hermitian line bundle since $f_{\varepsilon} \geqslant 0$. Furthermore, one has $\bar{M}_{\varepsilon, 1} \otimes \bar{M}_{\varepsilon, 2}^{\vee} \cong \overline{\mathcal{O}}\left(f_{\varepsilon}\right)$. Let $\bar{F}$ be an ample Hermitian line bundle on $X$ such that $\bar{F} \otimes \bar{L}^{\vee}$ is effective. One has

$$
\begin{array}{r}
\left\langle\widehat{c}_{1}(\bar{L})^{d}\right\rangle \cdot\left(\widehat{c}_{1}\left(\bar{M}_{\varepsilon, 2}\right)-\widehat{c}_{1}\left(\bar{M}_{\varepsilon, 1}\right)\right)=\left\langle\widehat{c}_{1}(\bar{L})^{d}\right\rangle \cdot \widehat{c}_{1}\left(\overline{\mathcal{O}}\left(f_{\varepsilon}\right)\right) \\
\leqslant\left\langle\widehat{c}_{1}(\bar{L})^{d}\right\rangle \cdot \widehat{c}_{1}(\overline{\mathcal{O}}(\varepsilon)) \leqslant \widehat{c}_{1}(\bar{F})^{d} \widehat{c}_{1}(\overline{\mathcal{O}}(\varepsilon))=\varepsilon \int_{X(\mathbb{C})} c_{1}(\bar{F})^{d},
\end{array}
$$

where in the first inequality, we have applied Proposition 3.10 (see also Remark 3.11), and in the second inequality, we have used the fact that $\overline{\mathcal{O}}(\varepsilon)$ is nef and then applied Lemme 3.7. Since $\varepsilon$ is arbitrary, we obtain that

$$
D_{\bar{L}} \widehat{\operatorname{vol}}(\bar{M}):=\lim _{n \rightarrow+\infty} \frac{\widehat{\operatorname{vol}}\left(\bar{L}^{\otimes n} \otimes \bar{M}\right)-\widehat{\operatorname{vol}}\left(\bar{L}^{\otimes n}\right)}{n^{d}}
$$


exists in $\mathbb{R}$. Since

$$
(d+1)\left\langle\widehat{c}_{1}(\bar{L})^{d}\right\rangle \cdot \widehat{c}_{1}\left(\bar{M}_{\varepsilon, 2}\right) \leqslant D_{\bar{L}} \widehat{\operatorname{vol}}(\bar{M}) \leqslant(d+1)\left\langle\widehat{c}_{1}(\bar{L})^{d}\right\rangle \cdot \widehat{c}_{1}\left(\bar{M}_{\varepsilon, 1}\right),
$$

by virtue of Remark 3.11 , we obtain $D_{\bar{L}} \widehat{\operatorname{vol}}(\bar{M})=(d+1)\left\langle\widehat{c}_{1}(\bar{L})^{d}\right\rangle \cdot \widehat{c}_{1}(\bar{M})$, which is additive with respect to $\bar{M}$.

A direct consequence of Theorem 1.1 is the asymptotic orthogonality of arithmetic Fujita approximation.

Corollary 4.3. - Assume that $\bar{L}$ is a big Hermitian line bundle on X. One has

$$
\left\langle\widehat{c}_{1}(\bar{L})^{d}\right\rangle \cdot \widehat{c}_{1}(\bar{L})=\widehat{\operatorname{vol}}(\bar{L}) \text {. }
$$

Proof. - By definition,

$$
\begin{aligned}
& D_{\bar{L}} \widehat{\operatorname{vol}}(\bar{L})=\lim _{n \rightarrow+\infty} \frac{\widehat{\operatorname{vol}}\left(\bar{L}^{n+1}\right)-\widehat{\operatorname{vol}}\left(\bar{L}^{\otimes n}\right)}{n^{d}} \\
= & \widehat{\operatorname{vol}}(\bar{L}) \lim _{n \rightarrow+\infty} \frac{(n+1)^{d+1}-n^{d+1}}{n^{d}}=(d+1) \widehat{\operatorname{vol}}(\bar{L}) .
\end{aligned}
$$

So (12) follows from Theorem 1.1 .

Remark 4.4. - As mentioned in Introduction, the differentiability of the geometrical volume function can be obtained by using the method of Okounkov bodies developed in $[\mathbf{2 3}$. See [18 for a proof of this result and other interesting results concerning geometric volume functions. Recently, Yuan [27] has proposed a partial analogue of the construction of Lazarsfeld and Musţată in Arakelov geometry. In fact, he has defined the Okounkov bodies of a Hermitian line bundle with respect to so-called "vertical flags". This permits him to obtain the log-concavity of the arithmetic volume function and the analogue of Fujita's approximation theorem in Arakelov goemetry, where the latter has also been independently obtained by the present author [12, using asymptotic measures. Quite possibly, an analogue of Lazarsfeld and Musţată's construction with respect to "horizontal flags" could also imply the differentiability of the arithmetic volume function.

\section{Applications and comparisons}

In this section, we shall apply our differentiability result to study several arithmetic invariants of Hermitian line bundles.

5.1. Asymptotic measure. - Let $\bar{L}$ be a Hermitian line bundle on $X$ such that $L_{K}$ is big. The asymptotic measure of $\bar{L}$ is the vague limit in the space of Borel probability measures

$$
\nu_{\bar{L}}:=-\lim _{n \rightarrow+\infty} \frac{\mathrm{d}}{\mathrm{d} t} \frac{\operatorname{rk}\left(\operatorname{Vect}_{K}\left(\left\{s \in \pi_{*} L^{\otimes n} \mid \forall \sigma,\|s\|_{\sigma, \text { sup }} \leqslant e^{-\lambda n}\right\}\right)\right)}{\operatorname{rk}\left(\pi_{*} L^{\otimes n}\right)},
$$

where the derivative is taken in the sense of distribution. It is also the limit of normalized Harder-Narasimhan measures (cf. [10, 11, 12]). 
Note that the support of the probability measure $\nu_{\bar{L}}$ is contained in $\left.]-\infty, \widehat{\mu}_{\max }^{\pi}(\bar{L})\right]$, where $\widehat{\mu}_{\max }^{\pi}(\bar{L})$ is the limit of maximal slopes (see [10, Theorem 4.1.8]):

$$
\widehat{\mu}_{\max }^{\pi}(\bar{L}):=\lim _{n \rightarrow+\infty} \frac{\widehat{\mu}_{\max }\left(\pi_{*} \bar{L}^{\otimes n}\right)}{n} .
$$

Recall that in [11, Theorem 5.5], the present author has proved that $\widehat{\mu}_{\max }^{\pi}(\bar{L})>0$ if and only if $L_{K}$ is big. Furthermore, by definition, one has $\widehat{\mu}_{\max }^{\pi}(\bar{L}(a))=\widehat{\mu}_{\max }^{\pi}(\bar{L})+a$ for any $a \in \mathbb{R}$. Therefore, $\widehat{\mu}_{\max }^{\pi}(\bar{L})$ is also the infimum of all real numbers $\varepsilon$ such that $\bar{L}(-\varepsilon)$ is big.

The asymptotic measure is a very general arithmetic invariant. Many arithmetic invariants of $\bar{L}$ can be represented as integrals with respect to $\nu_{\bar{L}}$. In the following, we discuss some examples. The asymptotic positive slope of $\bar{L}$ is defined as

$$
\widehat{\mu}_{+}^{\pi}(\bar{L}):=\frac{1}{[K: \mathbb{Q}]} \frac{\widehat{\operatorname{vol}}(\bar{L})}{(d+1) \operatorname{vol}\left(L_{K}\right)} .
$$

In [11, the author has proved that $\widehat{\mu}_{+}^{\pi}(\bar{L})$ is also the maximal value of the asymptotic Harder-Narasimhan polygon of $\bar{L}$ and that the asymptotic positive slope has the following integral form:

$$
\widehat{\mu}_{+}^{\pi}(\bar{L})=\int_{\mathbb{R}} \max (x, 0) \nu_{\bar{L}}(\mathrm{~d} x) .
$$

More generally, for any $a \in \mathbb{R}$, one has

$$
\int_{\mathbb{R}} \max (x-a, 0) \nu_{\bar{L}}(\mathrm{~d} x)=\widehat{\mu}_{+}^{\pi}(\bar{L}(-a)) .
$$

Another important example is the asymptotic slope of $\bar{L}$, which is

$$
\widehat{\mu}^{\pi}(\bar{L}):=\frac{1}{[K: \mathbb{Q}]} \frac{S(\bar{L})}{(d+1) \operatorname{vol}\left(L_{K}\right)} \in[-\infty,+\infty[,
$$

where $S(\bar{L})$ is the sectional capacity of $\bar{L}$ as in (2). The asymptotic slope has the following integral form

$$
\widehat{\mu}^{\pi}(\bar{L})=\int_{\mathbb{R}} x \nu_{\bar{L}}(\mathrm{~d} x) .
$$

Observe that we have

$$
\widehat{\mu}_{\max }^{\pi}(\bar{L}) \geqslant \widehat{\mu}_{+}^{\pi}(\bar{L}) \geqslant \widehat{\mu}^{\pi}(\bar{L}) .
$$

Using Theorem 1.1 and the differentiability of geometric volume function in [8], we prove that the asymptotic positive slope $\widehat{\mu}_{+}^{\pi}$ is differentiable and calculate its differential.

Proposition 5.1. - Assume that $\bar{L}$ is a big Hermitian line bundle on X. For any Hermitian line bundle $\bar{M}$, one has

$$
D_{\bar{L}} \widehat{\mu}_{+}^{\pi}(\bar{M}):=\lim _{n \rightarrow+\infty}\left(\widehat{\mu}_{+}^{\pi}\left(\bar{L}^{\otimes n} \otimes \bar{M}\right)-\widehat{\mu}_{+}^{\pi}\left(\bar{L}^{\otimes n}\right)\right)
$$


exists in $\mathbb{R}$. Furthermore, one has

$$
D_{\bar{L}} \widehat{\mu}_{+}^{\pi}(\bar{M}):=\frac{\left\langle\widehat{c}_{1}(\bar{L})^{d}\right\rangle \cdot \widehat{c}_{1}(\bar{M})}{[K: \mathbb{Q}] \operatorname{vol}\left(L_{K}\right)}-\frac{d\left\langle c_{1}\left(L_{K}\right)^{d-1}\right\rangle \cdot c_{1}\left(M_{K}\right)}{\operatorname{vol}\left(L_{K}\right)} \widehat{\mu}_{+}^{\pi}(\bar{L}),
$$

where $\left\langle c_{1}\left(L_{K}\right)^{d-1}\right\rangle \cdot c_{1}\left(M_{K}\right)$ is the geometric positive intersection product $([\mathbf{8}, \S 2])$.

Proof. - This is a direct consequence of Theorem 1.1 and $[\mathbf{8}$, Theorem A], where the latter asserts that

$$
\lim _{n \rightarrow+\infty} \frac{\operatorname{vol}\left(L_{K}^{\otimes n} \otimes M_{K}\right)-\operatorname{vol}\left(L_{K}^{\otimes n}\right)}{n^{d-1}}=d\left\langle c_{1}\left(L_{K}\right)^{d-1}\right\rangle \cdot c_{1}\left(M_{K}\right) .
$$

We then deduce from Proposition 5.1 the expression of the distribution function of the measure $\nu_{\bar{L}}$.

Proposition 5.2. - The distribution function $F_{\bar{L}}$ of $\nu_{\bar{L}}$ satisfies the equality

$$
\left.\left.F_{\bar{L}}(a):=\nu_{\bar{L}}(]-\infty, a\right]\right)=1-\frac{\left\langle\widehat{c}_{1}(\bar{L}(-a))^{d}\right\rangle \cdot \widehat{c}_{1}(\overline{\mathcal{O}}(1))}{[K: \mathbb{Q}] \operatorname{vol}\left(L_{K}\right)}, \quad a<\widehat{\mu}_{\max }^{\pi}(\bar{L}) .
$$

Proof. - One has

$$
F_{\bar{L}}(a)=1+\frac{\mathrm{d}}{\mathrm{d} a} \int_{\mathbb{R}} \max (x-a, 0) \nu(\mathrm{d} x)=1+\frac{\mathrm{d}}{\mathrm{d} a} \widehat{\mu}_{+}^{\pi}(\bar{L}(-a)) .
$$

By Proposition 5.1 one obtains

$$
\frac{\mathrm{d}}{\mathrm{d} a} \widehat{\mu}_{+}^{\pi}(\bar{L}(-a))=-\frac{\left\langle\widehat{c}_{1}(\bar{L}(-a))^{d}\right\rangle \cdot \widehat{c}_{1}(\overline{\mathcal{O}}(1))}{[K: \mathbb{Q}] \operatorname{vol}\left(L_{K}\right)} .
$$

Remark 5.3. - 1) Since the support of $\nu_{\bar{L}}$ is bounded from above by $\widehat{\mu}_{\max }(\bar{L})$, one has $F_{\bar{L}}(a)=1$ for $a \geqslant \widehat{\mu}_{\max }(\bar{L})$.

2) As a consequence of Proposition [5.2, one obtains that the function

$$
\frac{\left\langle\widehat{c}_{1}(\bar{L}(-a))^{d}\right\rangle \cdot \widehat{c}_{1}(\overline{\mathcal{O}}(1))}{[K: \mathbb{Q}] \operatorname{vol}\left(L_{K}\right)}
$$

is decreasing with respect to $a$ on $]-\infty, \widehat{\mu}_{\max }^{\pi}(\bar{L})[$, which is also implied by Lemma 3.7. Furthermore, this function takes values in $] 0,1]$, and converges to 1 when $a \rightarrow-\infty$.

3) Let $a \in]-\infty, \widehat{\mu}_{\max }^{\pi}(\bar{L})[$. The restriction of

$$
\frac{1}{[K: \mathbb{Q}] \operatorname{vol}\left(L_{K}\right)}\left\langle\widehat{c}_{1}(\bar{L}(-a))^{d}\right\rangle
$$

on $C^{0}(X(\mathbb{C})$ ) (considered as a subgroup of $\widehat{\operatorname{Pic}}(X)$ via the mapping $f \mapsto \mathcal{O}(f)$ ) is a positive linear functional, thus corresponds to a Radon measure on $X(\mathbb{C})$. Furthermore, by 1 ), its total mass is bounded from above by 1 , and converges to 1 when $a \rightarrow-\infty$. 
4) After Remark [3.9, we observe from Proposition 5.2 that the only possible discontinuous point of the distribution function $F_{\bar{L}}(a)$ is $a=\widehat{\mu}_{\max }^{\pi}(\bar{L})$.

As an application, we calculate the sectional capacity in terms of positive intersection product.

Corollary 5.4. - Let $\bar{L}$ be a Hermitian line bundle on $X$ such that $L_{K}$ is big. Let $A=\widehat{\mu}_{\max }^{\pi}(\bar{L})$. One has

$S(\bar{L})=(d+1) A \lim _{x \rightarrow A-}\left\langle\widehat{c}_{1}(\bar{L}(-x))^{d}\right\rangle \cdot \widehat{c}_{1}(\overline{\mathcal{O}}(1))-\int_{-\infty}^{A}(d+1) x \mathrm{~d}\left\langle\widehat{c}_{1}(\bar{L}(-x))^{d}\right\rangle \cdot \widehat{c}_{1}(\overline{\mathcal{O}}(1))$.

5.2. Lower bound of the positive intersection product. - Our differentiability result permits to obtain a lower bound for positive intersection products of the form $\left\langle\widehat{c}_{1}(\bar{L})^{d}\right\rangle \cdot \widehat{c}_{1}(\bar{M})$, where $\bar{L}$ is a big Hermitian line bundle on $X$ and $\bar{M}$ is an effective Hermitian lien bundle on $X$, by using the log-concavity of the arithmetic volume function proved in [27.

Proposition 5.5. - Let $\bar{L}$ and $\bar{M}$ be two Hermitian line bundles on X. Assume that $\bar{L}$ is big and $\bar{M}$ is effective. Then

$$
\left\langle\widehat{c}_{1}(\bar{L})^{d}\right\rangle \cdot \widehat{c}_{1}(\bar{M}) \geqslant \operatorname{vol}(\bar{L})^{\frac{d}{d+1}} \operatorname{vol}(\bar{M})^{\frac{1}{d+1}} .
$$

Proof. - Theorem 1.1 shows

$$
\lim _{n \rightarrow+\infty} \frac{\operatorname{vol}\left(\bar{L}^{\otimes n} \otimes \bar{M}\right)-\operatorname{vol}(\bar{M})}{n^{d}}=(d+1)\left\langle\widehat{c}_{1}(\bar{L})^{d}\right\rangle \cdot \widehat{c}_{1}(\bar{M}) .
$$

By [27, Theorem B], one has

$$
\widehat{\operatorname{vol}}\left(\bar{L}^{\otimes n} \otimes \bar{M}\right) \geqslant\left(\widehat{\operatorname{vol}}\left(\bar{L}^{\otimes n}\right)^{\frac{1}{d+1}}+\widehat{\operatorname{vol}}(\bar{M})^{\frac{1}{d+1}}\right)^{d+1} .
$$

By passing to limit, we obtain the required inequality.

Remark 5.6. - The inequality (17) could be considered as an analogue in Arakelov geometry (suggested by Bertrand [4]) of the isoperimetric inequality proved by Federer [13, 3.2.43]. See [14, §5.4] for an interpretation in terms of intersection theory, and [4. §1.2] for an analogue in geometry of numbers.

5.3. Comparison to other differentiability results. - We finally compare our results to several differentiability results on arithmetic invariants.

Intersection number. - Recall that the self-intersection number $\widehat{c}_{1}(\bar{L})^{d+1}$ is well defined for integrable Hermitian line bundles $\bar{L}$. See [15, 28, 29]. Furthermore, it is a polynomial function. Therefore, for any integrable Hermitian line bundles $\bar{L}$ and $\bar{M}$, one has

$$
\lim _{n \rightarrow+\infty} \frac{\widehat{c}_{1}\left(\bar{L}^{\otimes n} \otimes \bar{M}\right)^{d+1}-\widehat{c}_{1}\left(\bar{L}^{\otimes n}\right)}{n^{d}}=(d+1) \widehat{c}_{1}(\bar{L})^{d} \widehat{c}_{1}(\bar{M}) .
$$

This formula shows that the intersection number is differentiable at $\bar{L}$ along all directions in $\widehat{\operatorname{Int}}(X)$. 
Sectional capacity. - By using the analogue of Siu's inequality in Arakelov geometry, Yuan [26] has actually proved that the sectional capacity $S$ is differentiable along integrable directions at any Hermitian line bundle $\bar{L}$ such that $L$ is ample and that the metrics of $\bar{L}$ are semi-positive. Furthermore, for such $\bar{L}$, one has

$$
D_{\bar{L}} S(\bar{M}):=\lim _{n \rightarrow+\infty} \frac{S\left(\bar{L}^{\otimes n} \otimes \bar{M}\right)-S\left(\bar{L}^{\otimes n}\right)}{n^{d}}=(d+1) \widehat{c}_{1}(\bar{L})^{d} \widehat{c}_{1}(\bar{M}),
$$

where $\bar{M}$ is an arbitrary integrable Hermitian line bundles. This result has been established by Autissier [2] in the case where $d=1$. Recently Berman and Boucksom [3] have proved a general differentiability result for the sectional capacity. They have proved that the function $S$ is differentiable along the directions defined by continuous functions on $X(\mathbb{C})$ on the cone of generically big Hermitian lien bundles. Namely, for any continuous function $f$ on $X(\mathbb{C})$ and any Hermitian line bundle $\bar{L}$ on $X$ such that $L_{K}$ is big and that $S(\bar{L})$ is finite, the limit

$$
\lim _{n \rightarrow+\infty} \frac{S\left(\bar{L}^{\otimes n}(f)\right)-S\left(\bar{L}^{\otimes n}\right)}{n^{d}}
$$

exists. They have also computed explicitly the differential in terms of the MongeAmpère measure of $\bar{L}$ (see Theorem 5.7 and Remark 5.8 loc. cit.).

Our differentiability result for arithmetic volume function (Theorem 1.1), combined with (15), implies the differentiability of arithmetic invariants which can be written as the integration of a (fixed) smooth function of compact support with respect to the asymptotic measure of the Hermitian line bundle, by using integration by part. It would be interesting to know if a similar idea permits to deduce the differentiability of the sectional capacity, which can be written as the integral of the function $f(x)=x$ with respect to the asymptotic measure, along any direction at any Hermitian line bundle $\bar{L}$ such that $L_{K}$ is big and that $S(\bar{L})$ is finite.

\section{References}

[1] A. Abbes \& T. Bouche - Théorème de Hilbert-Samuel "arithmétique", Université de Grenoble. Annales de l'Institut Fourier 45 (1995), no. 2, p. 375-401.

[2] P. Autissier - Points entiers sur les surfaces arithmétiques., Journal für die Reine und Angewandte Mathematik 531 (2001), p. 201-235.

[3] R. Berman \& S. Boucksom - Capacities and weighted volumes of line bundles, 2008, arXiv:0803.1950.

[4] D. Bertrand - Minimal heights and polarizations on group varieties, Duke Mathematical Journal 80 (1995), no. 1, p. 223-250.

[5] J.-B. Bost - Périodes et isogénies des variétés abéliennes sur les corps de nombres (d'après D. Masser et G. Wüstholz), Astérisque (1996), no. 237, p. Exp. No. 795, 4, 115161, Séminaire Bourbaki, Vol. 1994/1995.

[6] J.-B. Bost, H. Gillet \& C. Soulé - Heights of projective varieties, Journal of the American Mathematical Society 7 (1994), no. 4, p. 903-1027.

[7] S. Boucksom, J.-P. Demailly, M. Paun \& T. Peternell - The pseudoeffective cone of a compact Käler manifold and varieties of negative Kodaira dimension, arXiv:math/0405285, 2004. 
[8] S. Boucksom, C. FAvre \& M. Jonsson - Differentiability of volumes of divisors and a problem of Teissier, 2006, to appear in Journal of algebraic geometry.

[9] A. Chambert-Loir \& A. Thuillier - Mesures de Mahler et équidistribution logarithmique, 2006, to appear in Annales de l'Institut Fourier.

[10] H. Chen - Convergence des polygones de Harder-Narasimhan, hal-00239438, 2007.

[11] _ Positive degree and arithmetic bigness, arXiv:0803.2583, 2008.

[12] Arithmetic Fujita approximation, arXiv:0810.5479, 2008.

[13] H. FEDERER - Geometric measure theory, Die Grundlehren der mathematischen Wissenschaften, Band 153, Springer-Verlag New York Inc., New York, 1969.

[14] W. Fulton - Introduction to toric varieties, Annals of Mathematics Studies, vol. 131, Princeton University Press, Princeton, NJ, 1993, The William H. Roever Lectures in Geometry.

[15] H. Gillet \& C. Soulé - Arithmetic intersection theory, Institut des Hautes Études Scientifiques. Publications Mathématiques (1990), no. 72, p. 93-174 (1991).

[16] _ An arithmetic Riemann-Roch theorem, Inventiones Mathematicae 110 (1992), no. 3 , p. $473-543$.

[17] R. LazArsfeld - Positivity in algebraic geometry. I, Ergebnisse der Mathematik und ihrer Grenzgebiete. 3. Folge. A Series of Modern Surveys in Mathematics, vol. 48, SpringerVerlag, Berlin, 2004, Classical setting: line bundles and linear series.

[18] R. Lazarsfeld \& M. Mustaţa - Convex bodies associated to linear series, arXiv:0805.4559, 2008.

[19] V. Maillot - Géométrie d'Arakelov des variétés toriques et fibrés en droites intégrables, Mémoires de la Société Mathématique de France. Nouvelle Série (2000), no. 80, p. vi +129 .

[20] A. MoriwaKi - Arithmetic height functions over finitely generated fields, Inventiones Mathematicae 140 (2000), no. 1, p. 101-142.

[21] Continuity of volumes on arithmetic varieties, To appear in Journal of Algebraic Geometry. arXiv:math/0612269v2, 2007.

[22] Continuous extension of arithmetic volumes, arXiv:0809.1129, 2008.

[23] A. OKounkov - Brunn-Minkowski inequality for multiplicities, Inventiones Mathematicae 125 (1996), no. 3, p. 405-411.

[24] H. Randriambololona - Métriques de sous-quotient et théorème de Hilbert-Samuel arithmétique pour les faisceaux cohérents, Journal für die Reine und Angewandte Mathematik 590 (2006), p. 67-88.

[25] R. Rumely, C. F. Lau \& R. VARley - Existence of the sectional capacity, Memoirs of the American Mathematical Society 145 (2000), no. 690, p. viii +130.

[26] X. YUAN - Big line bundles over arithmetic varieties, Inventiones Mathematicae 173 (2007), no. 3, p. 603-649.

[27] On volumes of arithmetic line bundles, 2008, arXiv:0811.0226.

[28] S. Zhang - Positive line bundles on arithmetic varieties, Journal of the American Mathematical Society 8 (1995), no. 1, p. 187-221.

[29] Small points and adelic metrics, Journal of Algebraic Geometry 4 (1995), no. 2, p. $281-300$.

September 15, 2021

Huayi Chen, Université Paris Diderot - Paris 7, Institut de mathématiques de Jussieu, case 247, 4 place Jussieu, 75252 Paris Cedex • E-mail : chenhuayi@math.jussieu.fr 